\title{
ON A SUBLATTICE OF THE LATTICE.OF NORMAL FITTING CLASSES
}

\author{
A. R. MAKAN
}

ABSTRACT. Let $L$ be the set of all Fitting classes $\mathcal{F}$ with the following two properties: (i) $\mathcal{F} \supseteq \boldsymbol{r}$, the class of all finite nilpotent groups, and (ii) every $\mathscr{F}$-avoided, complemented chief factor of any

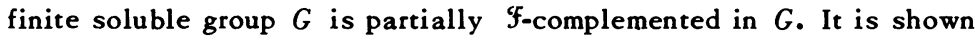
that $\mathbf{L}$ is a complete sublattice of the complete lattice $\mathrm{N}$ of all nontrivial normal Fitting classes, and, moreover, it is lattice isomorphic to the subgroup lattice of the Frattini factor group of a certain abelian torsion group due to $\mathrm{H}$. Lausch.

1. Introduction. All groups considered in this note are finite and soluble except when stated otherwise. A class $\mathcal{X}$ of groups is called a Fitting class if (1) $N \in X$ whenever $G \in X$ and $N \unlhd G$, and (2) $G \in X$ whenever $G$ is the product of normal subgroups each of which belongs to $X$. Given a Fitting class $\mathcal{F}$, a subgroup $V$ of a group $G$ is called an $\mathcal{F}$-injector of $G$ if, for any subnormal subgroup $N$ of $G, V \cap N$ is an $\mathcal{F}$-maximal subgroup of $N$. In view of Satz 1 of Fischer, Gaschütz and Hartley [3], every group $G$ has a unique conjugacy class of $\mathcal{F}$-injectors corresponding to every Fitting class $\mathcal{F}$. For various interesting properties of injectors we refer the readers to [3] and Hartley [5].

As in [8], a Fitting class $X$ is said to have the property $(\Lambda)$ if in each group $G$ every $X$-avoided, complemented chief factor of $G$ is necessarily a partially $X$-complemented chief factor. A chief factor of a group $G$ is called $X$-avoided $(X$-covered) if it is avoided (covered) by an $X$-injector of $G$. A complemented chief factor of $G$ is said to be partially $X$-complemented if at least one of its complements contains an $X$-injector of $G$. In [8], the author showed that a Fischer class (see Hartley [ 5$]$, for the definition) has the property $(\Lambda)$ if and only if it is the Fischer class of all solu-

Received by the editors August 30, 1973.

AMS (MOS) subject classifications (1970). Primary 20D25; Secondary 20D10, 20D30, 06A23.

Key words and phrases. Normal Fitting class, injector, normal Fitting pair, complete lattice, standard and twisted wreath products. 
ble $\pi$-groups for some set $\pi$ of primes. As pointed out there, one can have, in general, a Fitting class which has the property $(\Lambda)$ but which is not the class of all soluble $\pi$-groups for any set $\pi$ of primes. In fact, the (normal) Fitting class of all groups each of which acts by conjugation as an even permutation group on its largest normal 2 -subgroup is an example of such a Fitting class. For the details of this Fitting class, we refer the readers to Blessenohl and Gaschütz [2].

As in [2], a Fitting class $\mathfrak{F}$ is called normal if in every group $G$ an $\mathcal{F}$-injector of $G$ is a normal subgroup. In view of [2, Satz 6.2], the set $\mathbf{N}$ of all nontrivial normal Fitting classes is a complete lattice under the operations $\wedge$ and $\vee$ defined by

$$
\mathcal{F}_{1} \wedge \mathcal{F}_{2}=\mathcal{F}_{1} \cap \mathcal{F}_{2} \text { and } \mathcal{F}_{1} \vee \mathcal{F}_{2}=\bigcap\left\{\mathscr{X}_{\in} \mathbf{N} \mid \mathcal{F}_{1}, \mathcal{F}_{2} \subseteq \mathcal{X}\right\} \quad \forall \mathcal{F}_{1}, \mathcal{F}_{2} \in \mathbf{N}
$$

Recently, Lausch [7] has shown that this lattice $\mathbf{N}$ is lattice isomorphic to the subgroup lattice of a certain infinite abelian torsion group which he constructs in the course of proving Theorem 2.4 in [7]. More precisely, he shows (cf. [7, Corollary 2.5]) that if $(A, d)$ is a normal Fitting pair (see [7] for the definition) admitted by the least el ement of $\mathbf{N}$, then $\mathbf{N}$ is lattice isomorphic to the subgroup lattice of $A$. Our aim in this note is to prove the following:

Theorem 1. Let $\mathrm{L}$ be the set of all Fitting classes with the property $(\Lambda)$ each of which contains the class $\pi$ of all finite nilpotent groups. Then:

(1) $\mathbf{L}$ is a complete sublattice of $\mathbf{N}$; and

(2) if $(A, d)$ is a normal Fitting pair admitted by the least element of $\mathbf{N}$, then $\mathbf{L}$ is lattice isomorphic to the subgroup lattice of $A / \Phi(A)$, where $\Phi(A)$ denotes the Frattini subgroup of $A$.

For the definition of $\Phi(A)$, we refer the readers to Fuchs [4, p. 35]. The above theorem is proved in $\$ 3$, while in $\$ 2$ we discuss a property of the elements of $\mathbf{L}$ on which hinges the proof of Theorem 1.

2. Fitting classes with the property $(\Lambda)$. Our main result of this section is the following:

Theorem 2. Every Fitting class with the property $(\Lambda)$ which contains $r$ is normal.

In order to prove this theorem we need the following result which is an extension of Lemma 1 of [1]. Once again we refer the readers to Huppert [6] 
and B. H. Neumann [10] for the definitions of the standard and the twisted wreath products, respectively.

Lemma 3. Let $A$ and $B$ be two finite groups, $S \leq B$, a a homomorphism from $S$ into the automorphism group of $A$, and $T$ a right transversal of $S$ in $B$. Let $A \sim_{S} B=\left[A^{T}\right] B$ be the twisted wreath product of $A$ by $B$ over $S$. If $T=X C$, where $C$ is a subgroup of $B$ and $X$ is a subset of $a$ subgroup $R$ of $B$ such that $R \cap C=\{1\}$, then $\left[A^{T}\right] C \cong A^{X} \sim_{r} C$.

Proof. We observe first that if $f \in A^{T}$ then, for each $b \in B$ and each $t \in T, f^{b}(t)=a\left(s^{-1}\right)\left(f\left(t^{\prime}\right)\right)$, where $t^{\prime}$ and $s$ are determined by the equation $t b^{-1}=s t^{\prime}$. Now, let $\phi$ be the mapping from $\left[A^{T}\right] C$ into $A^{X} \sim_{r} C$ which assigns to each element $(f, c) \in\left[A^{T}\right] c$ the element $\left(f^{*}, c\right) \in A^{X} \sim$, $C$, where $f^{*}: C \rightarrow A^{X}$ is defined by $\left(f^{*}(d)\right)(x)=f(x d)$ for each $d \in C$ and each $x \in X$. Then $\phi$ is well defined and, as in the proof of Lemma 1 of [1], $\phi$ is a monomorphism. It remains to show that $\phi$ is onto. To see this, let $(g, c) \in A^{X} \sim_{r} C$ and let $t \in T$. Since $T=X C, X \subseteq R \leq B$ and $R \cap C$ $=\{1\}$, there exist unique elements $d \in C$ and $x \in X$ such that $t=x d$. Now, define a mapping $f \in A^{T}$ by $f(t)=(g(d))(x)$ for each $t \in T$. Then $\phi(f, c)$ $=(g, c)$, and so, $\phi$ is onto, as required.

We can now prove Theorem 2.

Proof of Theorem 2. Let $\mathcal{F}$ be a Fitting class with the property $(\Lambda)$ and let $\mathcal{F} \supseteq \Re$. In view of the main result in [9], it. will be sufficient to show that $(G \times \cdots \times G) \sim_{r} C \in \mathcal{F}$ ( $p$ factors in first term) whenever $G \in \mathcal{F}$ and $C=\langle c\rangle$ is a cyclic group of order $p$, a prime. Let $R=\langle x\rangle$ be a cyclic group of order $p^{2}$, let $H=C \times R$ and let $K$ be the subgroup of $H$ generated by $c x^{p}$. Let $W$ be the twisted wreath product of $G$ by $H$ over $K$ with the action of $K$ on $G$ being the trivial one. If $X=\left\{1, x, \cdots, x^{p-1}\right\}$ and $T=X C$, the complex product, then $T$ is a right transversal of $K$ in $H$ and, moreover, $W$ is the semidirect product of $G^{T}$ by $H$, with the action of $H$ on $G^{T}$ being as follows: For each $f \in G^{T}$, each $h \in H$ and each $t \in T, f^{h}(t)=\left(f\left(t^{\prime}\right)\right)$, where $t^{\prime} \in T$ is determined by the equation $t h^{-1}=k t^{\prime}$ with $k \in K$. Thus, since $H$ is abelian, $K$ acts trivially on the base group $G^{T}$. In particular, $G^{T} \times K$ is contained in the $\mathcal{F}$-injector $V$ of $W$. But then we must have that $G^{T} C / G^{T}$ is an $\mathfrak{F}$-covered chief factor of $W$, for, otherwise it would be an $\mathfrak{F}$-avoided, complemented chief factor of $W$ which is not partially $\mathcal{F}$-complemented in $W$ since $G^{T} K$ is not contained in any complement of $G^{T} C / G^{T}$ in $W$. Thus, $V \geq G^{T} C$, and so, $G^{T} C \in \mathcal{F}$ since $G^{T} C \triangleleft \triangleleft V$. However, by Lemma 3, $G^{T} C \cong G^{X} \sim, C$. Hence, since $G^{X}$ is isomorphic to a direct product of $p$ 
copies of $G$, it follows that $(G \times \cdots \times G) \sim_{r} C \in \mathcal{F}$ ( $p$ factors in first term) and the theorem is proved.

We conclude this section with the remark that for a Fitting class $\mathcal{F}$ with the property $(\Lambda)$ which does not necessarily contain $\pi$, one can show, using the same techniques as those used in the proofs of the main result in [9] and Theorem 2 above, that the normalizer in any group $G$ of an $\mathcal{F}$-injector of $G$ has index a $\pi^{\prime}$-number, where $\pi$ is the uniquely determined set of primes such that $\Re \cap \mathcal{S}_{\pi} \subseteq \mathcal{F} \subseteq \mathcal{S}_{\pi}$, the class of all soluble $\pi$-groups ( see Hartley $[5, \$ 3.3$, Remark 1])。

3. Lattice properties of $L$. In this section, we will prove Theorem 1 , our main result of this note. For the proof of the theorem we will need the following two lemmas of which the first one is a simple characterization of Fitting classes in $\mathbf{L}$.

Lemma 4. Let $\mathcal{F}$ be a Fitting class which contains $\Re$. Then $\mathcal{F} \in \mathbf{L}$ if and only if $\mathfrak{F}$ is normal and the factor group of every group $G$ with respect to its $\mathfrak{F}$-injector has elementary abelian Sylow subgroups.

Proof. Suppose first that $\mathcal{F} \in \mathbf{L}$. Then, by Theorem $2, \mathfrak{F}$ is normal, and so, in view of Satz 5.3 of Blessenohl and Gaschütz [2], the factor group of every group with respect to its $\mathcal{F}$-injector is abelian. Assume to the contrary that there exists a group $K$ whose factor group $K / V$ with respect to its $\mathcal{F}$ injector $V$ is not elementary abelian. Then, clearly $K$ has a subgroup $G$ such that $G>V$ and $G / V$ is cyclic of order $p^{2}$, for some prime $p$. Let $x \in G$ be such that $\langle V, x\rangle=G$, let $H=\langle y\rangle$ be a cyclic group of order $p$ and let $W=$ $G \times H$. Then $V \times H$ is the $\mathfrak{F}$-injector of $W$, and, therefore, $V\left\langle x^{p} y\right\rangle \notin \mathscr{F}$. In particular, $V\left\langle x^{p} y\right\rangle / V$ is an $\mathfrak{F}$-avoided chief factor of $W$ which is, moreover, complemented in $W$. However, it is easy to check that $V\left\langle x^{p} y\right\rangle / V$ is not partially $\mathcal{F}$-complemented in $W$, contrary to $\mathcal{F}$ having the property $(\Lambda)$. Hence, the assertion.

Suppose next that $\mathcal{F}$ is normal and that the factor group of every group

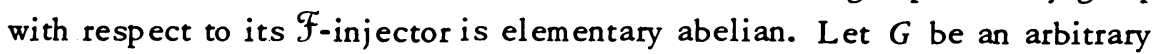
group, $V$ an $\mathcal{F}$-injector of $G$ and $H / K$ an $\mathcal{F}$-avoided chief factor of $G$. Since $G / V$ is elementary abelian, $V H / V K$ is clearly a complemented chief factor of $G$. But a complement of $V H / V K$ is also a complement of $H / K$ and, moreover, it contains $V$. Hence $\mathcal{F} \in \mathrm{L}$ and the proof is complete.

Next, we have

Lemma 5. Let $\left\{\mathcal{F}_{i} \mid i \in I\right\}$ be a family of Fitting classes. If $\mathfrak{F}_{i} \in \mathbf{L}$ for 
each $i \in I$, then $X=\bigcap_{i \in I} \mathcal{F}_{i} \in \mathbf{L}$. In particular, there exists a unique smallest Fitting class containing $\Re$ which has the property $(\Lambda)$.

Since each $\mathcal{F}_{i}$ is, by Theorem 2, a normal Fitting class, the above lemma follows from Blessenohl and Gaschütz [2, Lemma 4, Satz 6.2] and the fact

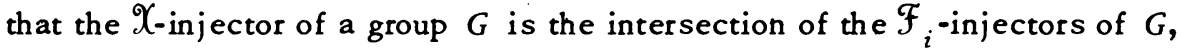
$i \in I$.

Finally, we can prove our main result.

Proof of Theorem 1. The first part of the theorem, namely that $L$ is a complete sublattice of $\mathbf{N}$, is immediate from Theorem 2 , Lemma 5 and the fact that $\mathfrak{F} \in \mathbf{L}$ implies $\mathcal{H} \in \mathbf{L}$ whenever $\mathcal{H}$ is a Fitting class such that $\mathfrak{F} \subseteq \mathcal{H}$. The latter fact follows easily from Lemma 4.

As for the second part of the theorem, let $\theta$ be the complete lattice isomorphism as defined in Lausch:[7, Corollary 2.5.] between $\mathbf{N}$ and the subgroup lattice of $A$, and let $(\theta \mid \mathbf{L})$ denote its restriction to the sublattice $\mathbf{L}$ of N. Set $\phi=(\theta \mid \mathbf{L}) \sigma$; where $\sigma: A \rightarrow A / \Phi(A)$ is the canonical epimorphism. Then $\phi$ is a complete lattice isomorphism from $L$ onto the subgroup lattice of $A / \Phi(A)$. For, observe first that if $\mathcal{F} \in \mathbf{L}$ then, as in the proof of Theorem 2.4 of Lausch [7], $(A / B, d \mu)$, where $B=\mathfrak{F}(\theta \mid \mathrm{L})$ and $\mu: A \rightarrow A / B$ is the canonical epimorphism, is a normal Fitting pair admitted by $\mathcal{F}$. Thus, in view of Lemma 4 and the definition of a normal Fitting pair, we have $\Phi(A) \subseteq B$. The rest follows from the fact that $\theta$ is a complete lattice isomorphism.

It follows from the proof of the second part of Theorem 1 and Proposition 2.1. of Lausch [7] that

Corollary 6. Let $\mathfrak{F} \in \mathbf{L}$ and let $\left(B, d_{1}\right)$ and $(A, d)$ be normal Fitting pairs admitted by $\mathcal{F}$ and the least element of $\mathbf{N}$, respectively. Then, $B$ is isomorphic to a factor group of $A / \Phi(A)$.

We conclude this note with a remark that $L$ is precisely the set of all Fitting classes each of which contains $\pi$ and has the property that in every group $G$ the corresponding injectors cover all the Frattini chief factors of $G$. For, let $\mathfrak{F} \supseteq \Re$ be a Fitting class with this property, let $G \in \mathcal{F}$ and let $C=$ $\langle c\rangle$ be a cyclic group of order $p^{2}$. If $W=G \sim, C=\left[G^{C}\right] C$, then clearly $\left[G^{C}\right]<c^{p}>\in \mathcal{F}$. But

$$
\begin{aligned}
{\left[G^{C}\right]\left\langle c^{p}\right\rangle \cong } & (G \times \cdots \times G) \sim \sim_{r} C_{p}, \\
\leftarrow p \text { factors } \rightarrow &
\end{aligned}
$$

where $C_{p}$ is a cyclic group of order $p$. Thus, $\mathscr{F}$ has the wreath product property, and so, by the main result of [9], $\mathcal{F}$ is a normal Fitting class. It is now easy to see that the factor group of every group $G$ with respect to its $\mathcal{F}$. 
injector is elementary abelian. Hence, $\mathcal{F} \in \mathbf{L}$. Conversely, if $\mathcal{F} \in \mathbf{L}$, then in view of Theorem 2 and Corollary 6 , the $\mathcal{F}$-injector of any group $G$ covers all the Frattini chief factors of $G$.

Acknowledgement. The author is indebted to the referee for several invaluable suggestions.

\section{REFERENCES}

1. J. C. Beidleman and A. R. Makan, On normal Fischer classes, Bull. London Math. Soc. 5 (1973), 100-102.

2. D. Blessenohl and W. Gaschütz, Über normale Schunck-und Fittingklassen, Math. Z. 118 (1970), 1-8. MR 43 \#3344.

3. B. Fischer, W. Gaschütz and B. Hartley, Injektoren endlicher auflös barer Gruppen, Math. Z. 102 (1967), 337-339. MR 36 \#6504.

4. L. Fuchs, Abelian groups, 3rd ed., Pergamon Press, London, New York, 1967.

5. B. Hartley, On Fischer's dualization of formation theory, Proc. London Math. Soc. (3) 19 (1969), 193-207. 39 \#5696.

6. B. Huppert, Endliche Gruppen. I, Die Grundlehren der Math. Wissenschaften, Band 134, Springer-Verlag, Berlin and New York, 1967. MR 37 \#302.

7. H. Lausch, On normal Fitting classes, Math. Z. 130 (1973), 67-72.

8. A. R. Makan, On a relation between injectors and certain complemented chief factors of finite soluble groups, J. Austral. Math. Soc. (to appear).

9. - Fitting classes with the wreath product property are normal, J. London Math. Soc. ( to appear).

10. B. H. Neumann, Twisted wreath products of groups, Arch. Math. 14 (1963), 1-6. MR $26 \# 5040$.

DEPARTMENT OF MATHEMATICS, UNIVERSITY OF KENTUCKY, LEXINGTON, KENTUCKY 40506 